\title{
One year outcomes after small incision lenticule extraction ReLEX in the correction of myopia and myopic astigmatism
}

\author{
Cristina Ariadna Nicula ${ }^{1,2}$, Dorin Nicula ${ }^{2}$, Sorana D. Bolboacă ${ }^{3 *}$ and Adriana Elena Bulboacă4
}

\begin{abstract}
Purpose: To report the visual and refractive outcomes of small incision lenticule extraction ReLEX (SMILE) technique using VisuMax femtosecond laser in myopia and myopic astigmatism patients.

Material and methods: A non-randomized clinical study has been conducted on patients with myopia and myopic astigmatism who underwent ReLEX SMILE technique, using the Zeiss VisuMax Laser system (Carl Zeiss Meditec AG, Jena, Germany) at Oculens Clinic, Cluj-Napoca, Romania. Patients older than 18 years, with ocular astigmatism up to -5 diopters (D), spherical equivalent up to -10.00 D, corrected distance visual acuity (CDVA) of 0.3 or better before the surgery, stable refraction for one year, and with a minimum calculated post operator residual stromal bed of $250 \mu$ were included in the study.
\end{abstract}

Results: The study involved a total of 25 myopic eyes (median of sphere diopters equal with -4D) and 67 myopic astigmatic eyes (median of cylinder diopters equal with -1.5 D). The mean refractive spherical equivalent (MRSE) on patients with myopic eyes reduced from -4.25D (median) to -0.5D at one month follow-up, $-0.25 \mathrm{D}$ at 6 and 12 months. The mean refractive spherical equivalent (MRSE) on patients with astigmatic myopic eyes reduced from6.25 $\mathrm{D}$ to $-0.67 \mathrm{D}$ at one month, $-0.62 \mathrm{D}$ at six and twelve months. The value of sphere decreased postoperatively on myopic eyes with a median of $-0.25 \mathrm{D}$ at one, six and twelve months. The value of cylinder decreased postoperatively on myopic astigmatic eyes with a median of $-0.50 \mathrm{D}$ at one month, $-0.25 \mathrm{D}$ at six months and $-0.50 \mathrm{D}$ at 12 months. At 6 and 12 months, 20 (80.0\%) of myopic eyes were maintained within $\pm 0.5 \mathrm{D}$ and 22 (88.0\%) with $\pm 1 \mathrm{D}$. On both groups (myopic eyes and myopic astigmatic eyes), statistically significant differences were observed when the keratometric baseline values were compared to each follow-up ( $P$-values $<0.0001)$, without any significant differences between follow-ups ( $P$-values $>0.15$ ). At 1-month follow-up, uncorrected distance visual acuity (UDVA) was better than or equal to 0.5 in $88.0 \%$ of myopic eyes and $82.1 \%$ of myopic astigmatic eyes. UDVA remained stable in all cases of myopic eyes at six months and the percentage increased at $92.0 \%$ in myopic eyes. UDVA slightly increased at 6-months (85.1\%) and remained at the same value at 12-months in myopic astigmatism eyes.

Conclusions: SMILE proved an effective and safe refractive corneal procedure and provided a predictable and stable correction of myopia and myopic astigmatism. SMILE technique demonstrated very good outcomes in terms of keratometric, cylinder, spherical measurements.

\footnotetext{
*Correspondence: sbolboaca@umfcluj.ro

${ }^{3}$ Department of Medical Informatics and Biostatistics, "Iuliu Hațieganu"

University of Medicine and Pharmacy, Louis Pasteur Str., no. 6,

400349 Cluj-Napoca, Romania

Full list of author information is available at the end of the article
} permits use, sharing, adaptation, distribution and reproduction in any medium or format, as long as you give appropriate credit to the original author(s) and the source, provide a link to the Creative Commons licence, and indicate if changes were made. The images or other third party material in this article are included in the article's Creative Commons licence, unless indicated otherwise in a credit line to the material. If material is not included in the article's Creative Commons licence and your intended use is not permitted by statutory regulation or exceeds the permitted use, you will need to obtain permission directly from the copyright holder. To view a copy of this licence, visit http://creativecommons.org/licenses/by/4.0/. The Creative Commons Public Domain Dedication waiver (http://creativeco mmons.org/publicdomain/zero/1.0/) applies to the data made available in this article, unless otherwise stated in a credit line to the data. 
Keywords: Myopia, Myopic astigmatism, Small Incision Lenticule Extraction (SMILE), Femtosecond laser

\section{Background}

Refractive lenticule extraction (ReLEX) includes small incision lenticule extraction (SMILE) and femtosecond lenticule extraction - the initial form of ReLEX (Flex). ReLEX is an alternative to keratomileusis in situ (LASIK) to correct myopia and myopic astigmatism done in 1990 for the first time by Pallikaris et al. [1]. Moreover, the technique is comparable to femtosecond laser-assisted in situ keratomileusis in terms of efficacy, safety, and predictability $[2,3]$. SMILE is becoming more and more popular as a flapless and minimally invasive form of laser vision correction for the treatment of myopia and myopic astigmatism using only one type of laser (femtosecond laser) for the entire surgery. This technology is only available in the VisuMax femtosecond laser platform (Carl Zeiss Meditec AG, Jena, Germany). The intervention with the femtosecond laser platform is minimally invasive and is the latest advancement in laser vision correction of myopia and myopic astigmatism.

Professor Walter Sekundo was the first who performed ReLEX in 2006. ReLEX SMILE is exclusively performed with one laser, a femtosecond laser that ensures highlevel reproducibility and predictability, even with high corrections [2-4]. The VisuMax system uses lower pulse energy and higher pulse frequency $(500 \mathrm{kHz})$. A low pulse energy is generally associated with fewer unwanted side effects (such as opaque bubble layer, collateral thermal damage, corneal inflammation) [4]. ReLEX SMILE has the advantages of better ocular surface stability and biomechanical strength compared with femto-LASIK techniques [4-7]. ReLEX SMILE provides a high quality of vision, the reason for being preferred in the treatment of myopia and myopic astigmatism, with an increase in patient satisfaction. During ReLEX SMILE, a VisuMax Femtosecond laser is used to create a disc of tissue called lenticule beneath the anterior surface of the cornea; the lenticule is then extracted through a small opening, eliminating the need for a flap.

Results of several studies reporting the outcomes after SMILE are summarized in Table 1 [8-23].

Previous studies have demonstrated that SMILE is safe and effective for the management of myopia and myopic astigmatism [13, 14, 16, 22]. The SMILE technique is applied in two centers in Romania, one in Bucharest and the other one in Cluj, at the Oculens Clinique. To the best of our knowledge, no study reporting SMILE safety and effectiveness on the Romanian population has been published in the scientific literature.

Table 1 Visual and refractive outcomes, safety and predictability of some recent studies performed on small incision lenticule extraction

\begin{tabular}{|c|c|c|c|c|c|c|}
\hline Study & No. of eyes & Duration, years & $\begin{array}{l}\text { Spherical } \\
\text { equivalent preop }\end{array}$ & $\begin{array}{l}\text { Spherical } \\
\text { equivalent postop }\end{array}$ & $\begin{array}{l}\text { Eyes within } \\
\pm 0.5 \mathrm{D}\end{array}$ & $\begin{array}{l}\text { UCVA (\%) } \\
-\geq 20 / \times \\
\text { postop }\end{array}$ \\
\hline Sekundo et al 2011 [8] & 91 & 0.5 & $-4.75 \pm 1.56$ & $-0.01 \pm 0.49$ & 80 & $84-20 / 20$ \\
\hline Shah et al 2011 [9] & 51 & 0.5 & $-4.87 \pm 2.16$ & $+0.03 \pm 0.30$ & 91 & $67-20 / 20$ \\
\hline Hjortdal et al 2012 [10] & 670 & 0.25 & $-7.19 \pm 1.30$ & $-0.25 \pm 0.44$ & 80 & $61-20 / 20$ \\
\hline Karmia et al 2014 [1 1] & 26 & 0.5 & $-4.21 \pm 1.63$ & +0.01 & 100 & $96-20 / 20$ \\
\hline Ağca et al 2014 [12] & 40 & 1 & $-4.03 \pm 1.61$ & $-0.02 \pm 0.06$ & 95 & $65-20 / 20$ \\
\hline Reinstein 2014 [13] & 110 & 1 & $-2.61 \pm 0.54$ & $-0.05 \pm 0.36$ & - & $96-20 / 20$ \\
\hline Vestergaard 2014 [14] & 127 & 0.25 & $-7.18 \pm 1.57$ & $-0.09 \pm 0.45$ & 77 & $\begin{array}{l}37-20 / 20 \\
73-20 / 25\end{array}$ \\
\hline Pedersen et al 2015 [15] & 87 & 3 & $-7.30 \pm 1.40$ & $-0.40 \pm 0.60$ & 70 & $49-20 / 25$ \\
\hline Chan et al 2015 [16] & 54 & 0.17 & $-6.05 \pm 1.46$ & $-0.10 \pm 0.23$ & 98.2 & $76-20 / 25$ \\
\hline Wu et al 2016 [17] & 39 & 1 & $-6.90 \pm 0.86$ & $-0.05 \pm 0.33$ & 96.7 & $93-20 / 25$ \\
\hline Blum et al 2016 [18] & 56 & 5 & $-4.89 \pm 4.97$ & -0.375 & 48.2 & n.a. \\
\hline Yildirim et al 2016 [19] & 45 & 2 & $-7.10 \pm 0.95$ & $-0.30 \pm 0.50$ & 92 & $86-20 / 20$ \\
\hline Han et al 2016 [20] & 47 & 2 & $-6.30 \pm 1.47$ & $-0.09 \pm 0.39$ & 89 & $92-20 / 20$ \\
\hline Liu et al 2016 [21] & 113 & 0.5 & $-5.22 \pm 1.70$ & $-0.03 \pm 0.13$ & 97 & $96-20 / 20$ \\
\hline Ganesh et al 2017 [22] & 50 & 0.25 & $-0.14 \pm 0.28$ & $-0.14 \pm 0.28$ & 86 & $96-20 / 20$ \\
\hline Pietila 2018 [23] & 300 & 1 & $-4.08 \pm 1.65$ & $0.04 \pm 0.48$ & 91 & $80-20 / 20$ \\
\hline
\end{tabular}


We aimed to report the visual and refractive outcomes of ReLEX Smile technique using VisuMax femtosecond laser in patients with myopia and myopic astigmatism at 12 months follow-up.

\section{Material and methods Subjects}

A non-randomized clinical study has been conducted on patients with myopia and myopic astigmatism who underwent ReLEX SMILE technique, using the Zeiss VisuMax Laser system (Carl Zeiss Meditec AG, Jena, Germany) at Oculens Clinic, Cluj-Napoca, Romania. All patients with ocular intervention from September 2018 to December 2018 were eligible for the study. Data were retrospectively collected in January 2020 from the medical charts after approval of the study by the ethics Oculens Clinic Ethics Committee (IRB 2018-004). The study was conducted by respecting the Declaration of Helsinki and extensive explanations regarding the procedure, possible intraoperative and postoperative complications. Written informed consent for treatment was obtained from all patients.

Patients older than 18 years, with ocular astigmatism up to -5.00 diopters (D), spherical equivalent up to $-10.00 \mathrm{D}$, corrected distance visual acuity (CDVA) of 0.3 or better before the surgery, stable refraction for one year, a minimum calculated post operator residual stromal bed of $250 \mu$, minimal preoperative pachymetry of $500 \mu \mathrm{m}$ were included in the study. No change in the keratometry data, cycloplegic refraction and axial length of the eye for one year were considered stability refractive criteria. Exclusion criteria were unstable refractive error, ectatic corneal disease (such as keratoconus, pellucid marginal degeneration), glaucoma, radial keratotomy or other previous ocular surgery, retinal degenerative disease, ocular trauma, uncontrolled ocular allergic disease, active blepharitis, significant dry eye, history of herpetic keratitis or any systemic disease that could affect wound healing (e.g., diabetes mellitus, systemic immunodeficiency, autoimmune disorders).

\section{Ocular examination}

A complete ocular assessment was performed prior to the laser correction to all patients. The following were collected for each patient included in the study: uncorrected distance visual acuity (UDVA) and corrected distance visual acuity (CDVA) were measured, refractometry (manifest and cycloplegic), keratometry (Topcon autorefracto-kerato-meter, KR 8900), slit lamp exam (Slit Lamp BX 900, Haag-Streit AG), eye fundus examination, intra-ocular pressure by applanation tonometry, ultrasonic pachymetry (Sonomed 300P Pachymeter), corneal tomography (Pentacam ${ }^{\circledR}$ HR Premium; Oculus
Optikgerate GmbH, Wetzlar, Germany), scotopic pupil size measurement with a pupilometer, ocular motor balance, dominance testing, fluorescein break-up time (TBUT) assessed with the slit lamp and endothelial cell counting (Konan SP-9000, Hyogo, Japan). Visual acuity was measured in the Snellen system using a projection chart at $5 \mathrm{~m}$. Patients were requested not to wear soft contact lenses for two weeks and rigid gas permeable ones for four weeks before evaluation or surgery.

Spherical equivalent refraction, cylinder, UDVA and CDVA, and keratometry values were measured before the surgery (baseline) and at 1, 6 and 12 months after the procedure.

\section{Surgical technique}

For all interventions, the VisuMax femtosecond laser system (Carl Zeiss Meditec, Jena, Germany) was used. The SMILE procedure was performed in the operating room in sterile conditions by the same surgeon. Topical anesthesia was used in all cases with 2-3 drops of oxibuprocaina solution (Benoxi, UNIMED PHARMA LTD, SLOVACIA) for 3 minutes.

Immediately prior to surgery, the lids were scrabbed with povidone-iodine solution (Betadine, EGIS Pharmaceuticals PLC, Budapesta, Hungary). An eyelid speculum was used to keep the eye wide open. A curved interface, which provided approximate alignment with the corneal surface, was used on the VisuMax femtosecond laser platform. The patient's eye was positioned under the curved contact glass of femtosecond laser. The suction was applied when the center of the pupil was centered on the contact lenses. Initially, the posterior surface of the refractive lenticule was created from the periphery to the center of the cornea. The anterior refractive lenticule was created from the center to the periphery and finally, the side cut was created at 12 o'clock position on a surface of $50^{\circ}$ length with the cordial length ranging from 2 to $5 \mathrm{~mm}$. After the laser treatment, a special SMILE double dissector (Dukeworth \& Kent, code 6-835) was used to break the remaining tissue bridges between the lenticule and stromal cap and those between the lenticule and stromal bed in order to make the lenticule freely extractable. Then the stromal lenticule was extracted with a special microforceps-SMILE lenticule removal (Dukeworth\&Kent, code 6-836E). The laser settings used for expert and standard mode are represented in Table 2 and the surgical parameters used during SMILE are given in Table 3.

The thickness of the lenticule was chosen according to the number of diopters to be corrected, considering that $15 \mu \mathrm{m}$ was necessary to correct one diopter.

All eyes aimed for the correction of emmetropia. We did not apply any manual compensation for 
Table 2 The settings of the laser

\begin{tabular}{ll}
\hline Parameters & Standard mode \\
\hline Frequency, $\mathrm{kHz}$ & $130-135$ \\
Energy offset, $(1$ offset $=5 \mathrm{~nJ})$ & $26-27$ \\
Spot distance, $\mu \mathrm{m}$ & \\
$\quad$ Lenticule and cap cuts & $4.2-4.3$ \\
$\quad$ Lenticule and cap side cuts & 2 \\
Track distance, $\mu \mathrm{m}$ & \\
$\quad$ Lenticule and cap cuts & $4.2-4.3$ \\
$\quad$ Lenticule and cap side cuts & 2 \\
\hline
\end{tabular}

Table 3 Surgical parameters applied in the SMILE technique

\begin{tabular}{|c|c|c|}
\hline Item & Lenticule & Cap \\
\hline Optical zone, mm & 6.5 & \\
\hline Thickness between, $\mu \mathrm{m}$ & 15 & $120-140$ \\
\hline Side cut angle, ${ }^{\circ}$ & 90 & 90 \\
\hline Transition zone, mm & 0.10 & \\
\hline Diameter, mm & 6.5 & 7.50 \\
\hline Incision position, $^{\circ}$ & & 90 \\
\hline Incision width, mm & & 3.93 \\
\hline
\end{tabular}

intraoperative cyclotorsion. All patients received at the end of the procedure one drop of Tobradex (Tobramicine and Dexamethasone) (NOVARTIS PHARMA GMBH - GERMANIA).

After the laser treatment, all patients underwent topical antibiotics and steroids (Tobramicine and Dexamethasone eye drops) five times daily for three days (NOVATIS PHARMA GMBH - GERMANIA), four times daily for one month with the tapered dose and artificial tears two times daily for 2 to 3 months. The patients were trained to avoid make-up and swimming for one week following the SMILE procedure. Slit-lamp examination was performed on $1^{\text {st }}$ and $4^{\text {th }}$ day, 1, 6 and 12 months after the surgery. Uncorrected distance visual acuity (UDVA) and corrected distance visual acuity (CDVA), manifest refraction, spherical equivalent (SE) and keratometry values were measured at 1, 6 and 12 months. Corneal topography was performed at 6 and 12 months postoperatively.

\section{Statistical analysis}

All raw data were collected using a Microsoft Excel spreadsheet (Microsoft Corporation, Redmond, WA, USA). The sample was split into two groups, myopic eyes and myopic astigmatic eyes. Collected data were presented as median and IQR (interquartile range defined as the first quartile to the third quartile) if quantitative (one group was with less than 30 eyes) or number (frequency) if qualitative. Differences between baseline and follow-ups were tested with the Friedman test at a significance level with Bonferroni correction (1.25\% for baseline, 1-month, 6-months, and 12-months follow-up; $1.67 \%$ for safety and efficacy index) followed by a posthoc analysis using Wilcoxon test. The safety index (SI) was defined as the (postoperative CDVA)/(CDVA at baseline). The efficacy index (EI) was defined as the (postoperative UDVA)/(CDVA at baseline).

The achieved correction versus the attempted refractive correction at 12 months was analysed with a linear regression model under the assumption of a normal distribution of refractive correction at 12 months. The association between the observed achievement (percentage of eyes within $\pm 0.50 \mathrm{D}$ ) at 6 -months and 12 -months was evaluated with Fisher's exact test at a significance level of $5 \%$. The association analysis between values of CDVA and keratometry measurements was evaluated with Spearman's rank correlation coefficient at a significance level of $5 \%$, and any correlation coefficient with $P$-values less than 0.05 were considered statistically significant.

Statistica program (v. 13, StatSoft, USA) was used for statistical analysis and all applied tests were two-tail.

\section{Results}

All eligible patients were systematically evaluated till 12 months postoperatively and were included in the analysis. A total of 92 eyes of 50 patients age from 19 to 63 years were evaluated, 25 myopic eyes (sphere diopters: median $=-4$, IQR $=(-4.5$ to -3$) ; 11 / 14$ patients with left and right myopic eyes and one patient with a myopic eye and a myopic astigmatic eye) and 67 myopic astigmatic eyes (cylinder diopters: median $=-1.5, \mathrm{IQR}=(-2.5$ to -1.0), MRSE: median $=-6.25, \mathrm{IQR}=(-7.25$ to -4.125$)$; $31 / 37$ with both eyes with myopic astigmatism). At baseline, the mean age of patients included in the analysis was $31.14 \pm 9.68$ years $($ median $=29.5$ years, $\mathrm{IQR}=(24$ to 32)), without significant differences of subjects with myopic eyes as compared to those with myopic astigmatic eyes (Mann-Whitney test: Z-statistics $=1.54$, $P$-value $=0.1224)$. The baseline parameters are shown in Table 4. The follow-up period was 12 months and all patients completed all follow-ups (one, six, and twelve months).

\section{Refractive outcomes: stability and predictability}

Refractive stability reproduces the changes in the mean refractive spherical equivalent (MRSE) error after SMILE (Fig. 1). The mean refractive spherical equivalent (MRSE) in patients with myopic eyes reduced from -4.25 $\mathrm{D}$ (median) $(\mathrm{IQR}=(-5.25$ to -3.12$))$ to $-0.5 \mathrm{D}(\mathrm{IQR}=$ $(-0.75$ to -0.25$))$ at 1 month, with a median value of -0.25 $\mathrm{D}(\mathrm{IQR}=(-1$ to -0.25$))$ at 6 months and $-0.25 \mathrm{D}(\mathrm{IQR}=$ $(-1$ to -0.25$))$ at 12 months. Friedman ANOVA showed 
Table 4 Baseline parameters of the group

\begin{tabular}{ll}
\hline Parameter & Value \\
\hline Gender, $\%$ & \\
Women & 57.60 \\
Men & 42.40 \\
Spheric equivalent, diopter ${ }^{\text {a }}$ & \\
$\quad$ Myopic eyes & $-4.25(-5.25$ to -3.12$)$ \\
Myopic astigmatic eyes & $-6.25(-7.25$ to -4.13$)$ \\
Statistic (P-value) & $2.97(0.0031)$ \\
Mean Keratometry, diopter ${ }^{\text {a }}$ & \\
$\quad$ Myopic eyes & $43.50(42.75$ to 44.85$)$ \\
$\quad$ Myopic astigmatic eyes & $44.20(43.10$ to 45.00$)$ \\
Statistic (P-value) & $0.66(0.5103)$ \\
\hline
\end{tabular}

a values are expressed as the median and interquartile range (Q1 to Q3), where Q1 is the first quartile and Q3 is the third quartile

statistically differences among follow-up examination (Statistics $=72.13, P$-value $<0.0001)$, with statistically significance differences when the baseline is compared with each follow-up $(P$-values $=0.00001)$. Furthermore, no significant differences were observed when 1-month follow-up was compared to 12 months follow-up (Wilcoxon pairs test: Statistics $=1.78, P$-value $=0.0754$ ) or 6 months follow-up was compared to 12 months follow-up (Wilcoxon pairs test: Statistics $=0.53, P$-value $=0.5930$ ).

The mean refractive spherical equivalent (MRSE) on patients with astigmatic myopic eyes reduced from -6.25 $\mathrm{D}$ (median) $(\mathrm{IQR}=(-7.25$ to -4.13$))$ to $-0.67 \mathrm{D}(\mathrm{IQR}=$ $(-1$ to -0.25$))$ at 1 month, with a median value of $-0.62 \mathrm{D}$ $(\mathrm{IQR}=(-1.13$ to -0.25$))$ at 6 months and $-0.62 \mathrm{D}$ (IQR $=(-1$ to -0.25$))$ at 12 months. Friedman showed statistically differences among follow-up examination (Statistics $=178.96, P$-value $<0.0001$ ), with statistically significance differences when the baseline is compared with each follow-up $(P$-values $=0.00001)$. Furthermore, no significant differences were observed when 1-month follow-up was compared to 12 months follow-up (Wilcoxon pairs test: Statistics $=1.34, P$-value $=0.1773$ ) or 6 months followup was compared to 12 months follow-up (Wilcoxon pairs test: Statistics $=0.53, P$-value $=0.5930$ ) .

The value of sphere decreased postoperatively on myopic eyes with a median of $-0.25 \mathrm{D}$ (IQR $=(-0.75$ to $0.00)$ ) at 1 month, $-0.25 \mathrm{D}(\mathrm{IQR}=(-0.50$ to -0.25$)$ at 6 months and $-0.25 \mathrm{D}(\mathrm{IQR}=(-0.50$ to 0.00$)$ at 12 months. Friedman test showed significant differences among follow-ups (Statistic $=62.02, P$-value $<0.0001)$. The postoperative mean sphere proved significantly smaller values than baseline for each investigated follow-up (Wilcoxon test: $P$-values $=0.00001)$. Furthermore, no significant differences were identified when paired follow-ups were compared (Wilcoxon test: $P$-values $>0.05$ ).

The value of cylinder decreased postoperatively on myopic astigmatic eyes with a median of $-0.50 \mathrm{D}$ (IQR $=(-0.75$ to 0.00$))$ at 1 month, $-0.25 \mathrm{D}$ (IQR $=(-0.75$ to $-0.25)$ at 6 months and $-0.50 \mathrm{D}(\mathrm{IQR}=(-0.75$ to 0.00$)$ at 12 months. Friedman test showed significant differences among follow-ups (Statistic $=138.32, P$-value $<0.0001$ ). The postoperative mean sphere proved significantly smaller values than baseline for each investigated followup (Wilcoxon test: $P$-values $<0.00001)$. Furthermore, no significant differences were identified when paired follow-ups were compared (Wilcoxon test: $P$-values $>0.10$ ), with an identical value of the cylinder at 6 and respectively 12 months for all eyes.

\section{Predictability}

At six months, $20(80.0 \%)$ of myopic eyes were maintained within $\pm 0.5 \mathrm{D}$ and $22(88.0 \%)$ within $\pm 1 \mathrm{D}$. At 12 months 20 (80.0\%), the myopic eyes were maintained within $\pm 0.5 \mathrm{D}$ cases and $22(88.0 \%)$ within $\pm 1 \mathrm{D}$. The percentage of eyes within $\pm 0.50 \mathrm{D}$ showed no significant difference between 6 months and 12 months follow-up

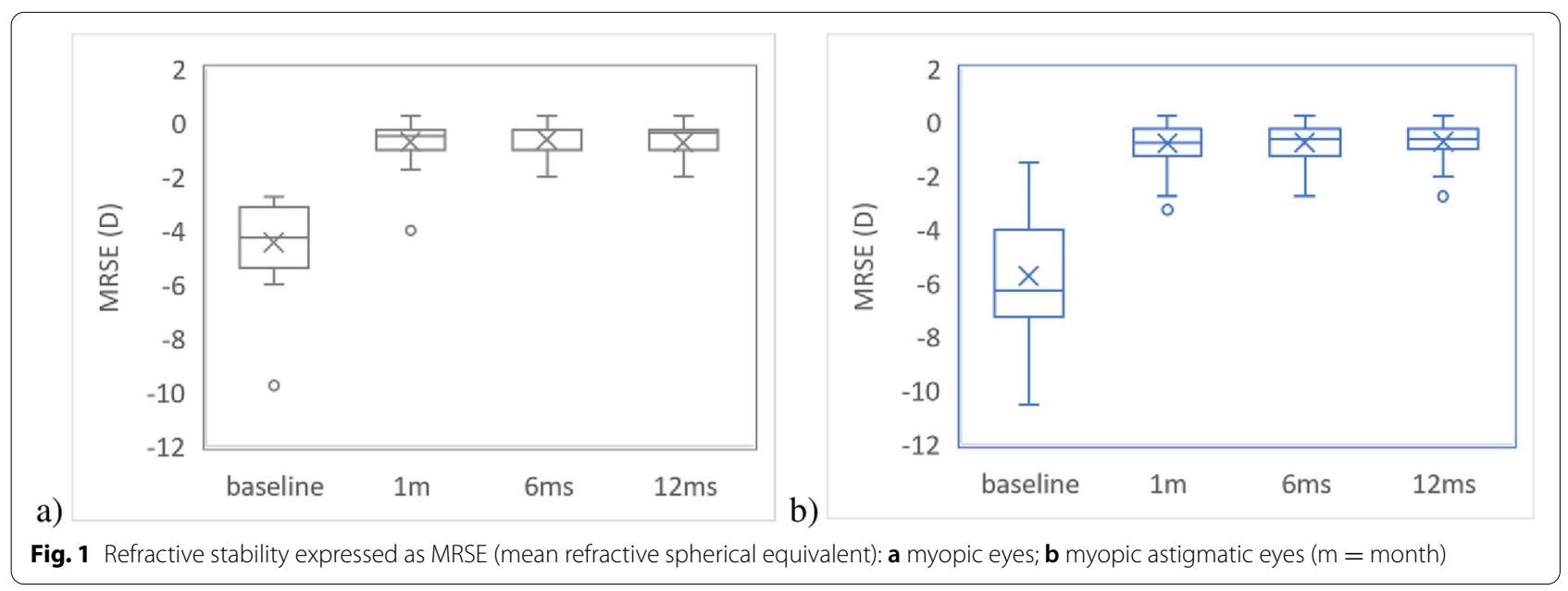


(Fisher exact test: $P$-value $>0.9999)$. At six months, 29 (43.3\%) of myopic astigmatic eyes were maintained within $\pm 0.5 \mathrm{D}$ and 48 (71.6\%) within $\pm 1 \mathrm{D}$. At 12 months 29 (43.3\%), the myopic astigmatic eyes were maintained within $\pm 0.5 \mathrm{D}$ cases and 49 (73.1\%) within $\pm 1 \mathrm{D}$. The percentage of eyes, both myopic and myopic astigmatic eyes, within $\pm 0.50 \mathrm{D}$ showed no significant difference between 6 months and 12 months follow-up ( $P$-values $>0.9999)$.

The association between the achieved and the attempted refractive correction at 12 months after the procedure is presented in Fig. 2.

The Kflat, Ksteep, and Kmean values decreased significantly one month postoperatively and showed similar values at 12 months follow-up on both groups ( $P$-values $<0.0001$ ). On both groups (myopic eyes and myopic astigmatic eyes), statistically significant differences were observed when the baseline values were compared to each follow-up (Wilcoxon matched test; $P$-values <
0.00002), without any significant differences between follow-ups (Wilcoxon matched test; $P$-value $>0.15$ ).

\section{Functional outcomes: safety and efficacy}

All eyes had a CDVA of 0.3 or better at baseline. At 1-month follow-up, UDVA was better than or equal to 0.5 in $88.0 \%$ of myopic eyes and $82.1 \%$ of myopic astigmatic eyes. UDVA remained stable in all cases of myopic eyes at 6-months, and the percentage increased to $92.0 \%$ on myopic eyes. The percentage of UDVA achievement slightly increased at 6-months (85.1\%) and remained at the same value at 12 -months on myopic astigmatism eyes.

The visual recovery indicates no changes in the UDVA and CDVA between 6-months and 12-months with time after the SMILE surgery, with significantly better 12-months results than baseline (Table 5).

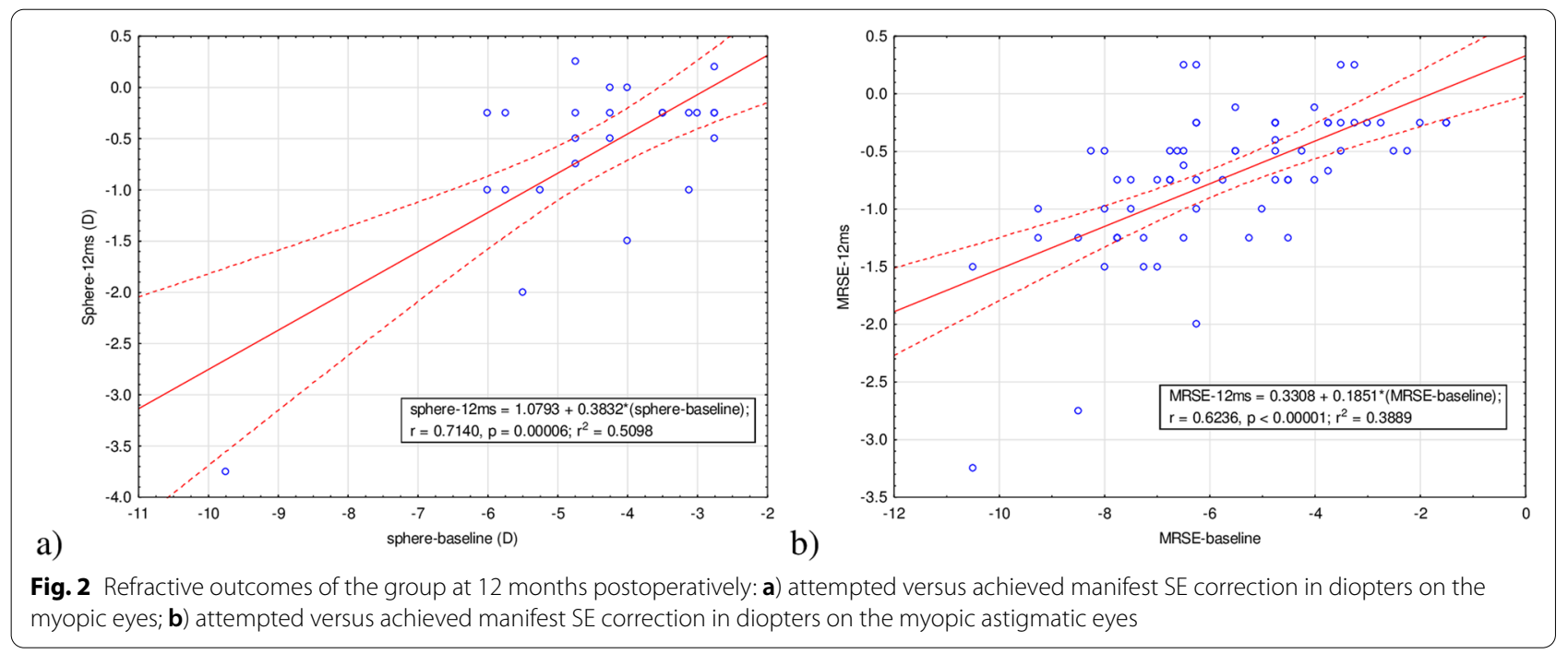

Table 5 UDVA and CDVA changes in time: comparisons between baseline and each follow-up

\begin{tabular}{|c|c|c|c|c|}
\hline & \multicolumn{2}{|l|}{$\begin{array}{l}\text { Myopic eyes } \\
n=25\end{array}$} & \multicolumn{2}{|c|}{$\begin{array}{l}\text { Myopic astigmatic eyes } \\
n=67\end{array}$} \\
\hline & Median (Q1 to Q3) & Stat. ( $p$-value) & Median (Q1 to Q3) & Stat. ( $P$-value) \\
\hline \multicolumn{5}{|l|}{ UDVA } \\
\hline Baseline & $0.10(0.10$ to 0.20$)$ & & 0.10 (0.05 to 0.10$)$ & \\
\hline $\begin{array}{l}1 \text { month } \\
6 \text { months } \\
12 \text { months }\end{array}$ & $\begin{array}{l}0.90(0.60 \text { to } 1.00) \\
0.90(0.80 \text { to } 1.00) \\
0.90(0.80 \text { to } 1.00)\end{array}$ & $\begin{array}{l}4.20(0.00003) \\
4.20(0.00003) \\
4.20(0.00003)\end{array}$ & $\begin{array}{l}0.80(0.50 \text { to } 0.90) \\
0.80(0.60 \text { to } 0.90) \\
0.80(0.55 \text { to } 0.90)\end{array}$ & $\begin{array}{l}7.12(<0.00001) \\
7.12(<0.00001) \\
7.12(<0.00001)\end{array}$ \\
\hline \multicolumn{5}{|l|}{ CDVA } \\
\hline Baseline & 1.00 (0.90 to 1.00$)$ & & $0.90(0.70$ to 1.00$)$ & \\
\hline $\begin{array}{l}1 \text { month } \\
6 \text { months } \\
12 \text { months }\end{array}$ & $\begin{array}{l}0.90(0.00 \text { to } 1.00) \\
0.90(0.00 \text { to } 1.00) \\
0.90(0.00 \text { to } 1.00)\end{array}$ & $\begin{array}{l}2.75(0.0060) \\
2.75(0.0060) \\
2.75(0.0060)\end{array}$ & $\begin{array}{l}0.40(0.00 \text { to } 0.90) \\
0.40(0.00 \text { to } 0.90) \\
0.40(0.00 \text { to } 0.90)\end{array}$ & $\begin{array}{l}5.06(<0.00001) \\
5.05(<0.00001) \\
4.95(<0.00001)\end{array}$ \\
\hline
\end{tabular}

Q1 first quartile, Q3 third quartile; Comparisons between baseline and each follow-up was done with Wilcoxon test 
The values of the safety and efficacy index are presented in Table 6.

No significant changes in the safety index was observed in myopic eyes $(P$-value $>0.9999)$ and in myopic astigmatic eyes $(P$-value $=0.1146)$. The Friedman test identified significant differences in efficacy index in myopic eyes (Statistic $=12.67, p=0.0018$ ) as well as in myopic astigmatic eyes (Statistic $=20.92, p=0.00003$ ). The significant differences were observed when EI at 1 month was compared to EI at 6-months (Wilcoxon Matched Pairs Test: Statistic $=2.43, P$-value $=0.0152, n=9)$ and EI at 12-months (Wilcoxon Matched Pairs Test: Statistic $=2.76, P$-value $=0.0058, n=11)$ on myopic eyes. Similarly, significant differences were observed when EI at 1 month was compared to EI at 6-months (Wilcoxon Matched Pairs Test: Statistic $=3.06, P$-value $=0.0022$, $n=12$ ) and EI at 12-months (Wilcoxon Matched Pairs Test: Statistic $=3.30, P$-value $=0.0010, n=14)$ on myopic astigmatic eyes. No significant differences were observed neither on myopic eyes (Wilcoxon Matched Pairs Test: Statistic $=2.02, P$-value $=0.0431, n=5)$, not on myopic astigmatic eyes (Wilcoxon Matched Pairs Test: Statistic $=0.53, P$-value $=0.5940, n=9)$ when the EI at 6-months was compared to EI at 12-months.

Positive monotonic association was identified just in myopic eyes between CDVA- $6 \mathrm{~m}$ and Kflat- $6 \mathrm{~m}$ (where $6 \mathrm{~m}$ $=6$-months $)-\rho=0.46(P$-value $=0.0212)$ - and CDVA$6 \mathrm{~m}$ and Kmean-6m $-\rho=0.45(P$-value $=0.0237)$. Similarly, association between CDVA-12m and Kflat-12m ( $\rho$ $=0.46, P$-value $=0.0212)$ and CDVA- $12 \mathrm{~m} \&$ Kmean $12 \mathrm{~m}$ ( $\rho=0.45, P$-value $=0.0237)$ in myopic eyes.

Complications were recorded in $22.82 \%$ eyes, which included a minor epithelial abrasion in ten eyes (9.2\%), small tears at the incision in 8 eyes $(8.69 \%)$ and some difficulties removing the lenticule in two eyes (2.17\%). In one eye, we had a lenticule remnant left in the interface, probably due to incorrect tearing of the lenticule resulting in irregular astigmatism. First of all, we performed an anterior segment ocular coherence tomography to localize the remnant lenticule. After one week, we reoperate, ending with the extraction of the lenticule and visual acuity recovery. No threatening visual complication was recorded and none of the patients showed signs of ectasia at 12 months follow-up. No patient complained about episodes of blurring vision or fluctuation of vision. None of the patients lost lines in CDVA postoperatively.

\section{Discussions}

This is a retrospective, non-randomised study that presented results at 12 months after ReLEX SMILE technique in low and moderate myopia and myopic astigmatism. ReLEX SMILE was used in our study to correct myopia up to $-9 \mathrm{D}$ and myopic astigmatism up to $-5 \mathrm{D}$. In our study, we demonstrated a statistically significant decrease of MRSE between baseline and all the followups $(P$-value $=0.00001)$ in myopic and astigmatic myopic eyes. Furthermore, no significant differences were seen comparing the 1 month follow-up with 12 months follow-up. Similar results showed Han et al. [20] in their study of 4 years refractive outcomes, wavefront aberrations and quality of life after SMILE for moderate-tohigh myopia (mean SE - $6.30 \pm 1.47 \mathrm{D}$ ) concluding that SMILE provides a predictable and stable correction, with no significant changes of MRSE during postoperative follow-ups up to 4 years. Similarly, Blum et al [18] reported no significant change of MRSE from a 6-month follow-up to 5-year postoperatively. In the same study, a mild regression of $-0.48 \mathrm{D}$ was observed over a period of 5 years [18] as a consequence of eye globe growth rather than a true progression at the corneal level. Wu et al. [17] compared the efficacy and stability of SMILE technique in high myopia versus moderate myopia, concluding that the rate of regression was statistically significant in high myopia compared with low or moderate myopia due to the increased keratocyte activation present in high myopic correction after the procedure.

Our SMILE results reported a high level of refractive predictability. At 12 months, $80 \%$ of myopic eyes were within $\pm 0.5 \mathrm{D}$ from the target and $88 \%$ were within \pm 1 D. At 12 months $43.3 \%$, the myopic astigmatic eyes

Table 6 The safety index and efficacy index expressed as the median and interquartile range (first quartile to the third quartile)

\begin{tabular}{llll}
\hline Index & $\begin{array}{l}\text { Myopic eyes } \\
\boldsymbol{n = 2 5}\end{array}$ & $\begin{array}{l}\text { Myopic astigmatic eyes } \\
\boldsymbol{n = 6 7}\end{array}$ & $\begin{array}{l}\text { Mann-Whitney test } \\
(\boldsymbol{P} \text {-value })\end{array}$ \\
\hline $\begin{array}{l}\text { Safety index (SI) } \\
1 \text { month }\end{array}$ & $0.90(0.00$ to 1.00$)$ & $0.71(0.00$ to 1.00$)$ & $0.59(0.5536)$ \\
6 months & $0.90(0.00$ to 1.00$)$ & $0.80(0.00$ to 1.00$)$ & $0.60(0.5506)$ \\
12 months & $0.90(0.00$ to 1.00$)$ & $0.80(0.00$ to 1.00$)$ & $0.43(0.6640)$ \\
Efficacy index (EI) & & & \\
1 month & $1.00(0.60$ to 1.00$)$ & $1.00(0.76$ to 1.00$)$ & $-0.80(0.4245)$ \\
6 months & $1.00(0.80$ to 1.00$)$ & $1.00(0.83$ to 1.00$)$ & $-0.95(0.3432)$ \\
12 months & $1.00(0.80$ to 1.00$)$ & $1.00(0.83$ to 1.00$)$ & $-0.80(0.4245)$ \\
\hline
\end{tabular}


were maintained within $\pm 0.5 \mathrm{D}$ cases and $73.1 \%$ within $\pm 1 \mathrm{D}$. Our results are comparable with refractive predictability reported in the literature $[8,14,24]$. Sekundo et al. [8] reported that $92 \%$ of the eyes were within $\pm 0.50 \mathrm{D}$ at one year after SMILE procedure. Ağca et al. [12] showed similar results in $95 \%$ of cases. The lower results on myopic eyes in our study than the results reported by Sekundo et al. [8] and Ağca et al. [12] could be explained by the presence of patients younger than 25 years in our sample on who we performed an extra correction of 0.25-0.5 D. Moshirfar et al. [25] demonstrated that SMILE technique is safe and predictable.

In our study, the safety index at 6 and 12 months was 0.9. Similar results were shown by $\mathrm{Ng}$ et al. [24], who obtained a safety index of 1.00 at 3 and 6 months after SMILE and Ivarsen et al. [26] demonstrated a safety index of 1.05 at 3 months after SMILE. Chan et al. [16] in their study of 54 myopic eyes operated with SMILE reported a safety index of 0.97 , with $7.4 \%$ of cases that lost one line in CDVA.

At one month follow-up, we obtained an UDVA equal or better than 0.5 in $88 \%$ of myopic eyes and $82.1 \%$ in myopic astigmatic eyes. At 12 months in $92 \%$ of myopic eyes and $85.1 \%$ of myopic astigmatic eyes, the UDVA was equal or better than 0.6. The efficacy index at all follow-ups was 1 in both groups. Similar results were shown by Pedersen et al. [15], Chan et al. [16] and Wu et al. [17]. Agca et al. [27] showed that the mean postoperative UDVA and CDVA achieved at 5 years of follow-up was $0.04 \pm 0.09 \operatorname{logMAR}$ and $0.00 \pm 0.04 \log$ MAR, respectively.

The efficacy of SMILE shown above depends significantly on the precision of the lenticule creation by the femtosecond laser. Reinstein et al used a very high-frequency digital ultrasound to measure the accuracy of the thickness of the SMILE lenticule and found that the readout central lenticule depth was $8.2 \mu \mathrm{m}$ thicker on average than the Artemis measured stromal thickness change. This difference was partially explained by alignment errors between the pre- and postoperative scans and partly by central stromal expansion caused by biomechanical changes occurring after SMILE [28].

Regarding important complications, we had a difficult dissection and extraction of the lenticule in two eyes $(2.17 \%)$ with low myopia. Some studies showed that during the learning curve, this complication was encountered to be present in $16 \%$ of cases [29,30]. To solve the problem is important to identify the correct dissection plane in order to extract the lenticule by using the anterior segment ocular coherence tomography. Meniscus sign (the meniscus-shaped gap between the diameter of lenticule gap and lenticule edge) [31], Shimmer sign (bright reflex around the dissecting instrument) [32] and white ring sign (light reflex from the lenticular side cut) [33] were used to identify the correct dissection plane.

Other complications in our study were recorded in $22.82 \%$ eyes, which included a minor epithelial abrasion in ten eyes $(9.2 \%)$, small tears at the incision in 8 eyes (8.69\%). Ivarsen et al. [26], in a study on 1800 eyes, reported an incidence of $6 \%$ of epithelial abrasions, $1.8 \%$ of small incision tears, $1.9 \%$ difficult lenticule extraction, $0.22 \%$ cap perforation and $0.06 \%$ of major tear. In cases of lenticule remnant being left in the interface, Ganesh et al. [34] propose for reevaluation of the anterior segment optical coherence tomography to establish the location of the lenticule. Ganesh et al. [34] concluded that retained pieces of lenticules can be extracted as late as 9 months of the failed SMILE procedure.

In our study, we did not report any ectasia. Moshirfar et al. [25], in their review upon ectasia after 750,000 SMILE procedures performed worldwide, reported only seven eyes with ectasia. The explanation of ectasia was that these cases were undiagnosed fruste keratoconus [35] or had an untrustworthy preoperative corneal topography [36].

In our study, we did not have any cases with severe blurring vision after SMILE. Some studies demonstrated a delay in the visual recovery and visual fluctuations with episodes of blurring of vision in the early postoperative period after SMILE compared to LASIK $[37,38]$. This might be as a result of suboptimal laser photo-disruption of the stromal fibers that further causes interface scattering due to traumatic dissection [39], micro-distortions in Bowman's layer after lenticule removal [40], and transient increased stromal keratocyte activity at the interface resulting from the stromal pocket irrigation [41].

Our study has several limitations that should be acknowledged. This was an observational single center retrospective study, and thus there was no random treatment allocation. However, the inter-physician variability was withdrawn in our study since only one surgeon treated all patients, but we did not control patients' variability since all eligible subjects were included in the study. The follow-up of our patients' was limited to one year, but the late follow-up (e.g., 2 years, 5 years, etc.) would also be clinically relevant in the assessment of the longterm effectiveness of this technique. A clinical trial with a large sample (multicenter) and an extended follow-up would confirm the validity of the reported results and the long-term effectiveness. To our knowledge, this is the first study regarding the SMILE technique done in Romania, so our results could be used as input data in sample size calculations on our population. 


\section{Conclusions}

SMILE proved an effective and safe refractive procedure and provided a predictable and stable correction of low and moderate myopia and myopic astigmatism. The 12-months results showed that it is a very efficient procedure, with few complications and good visual outcomes. The long-term follow-up would bring clinically relevant information regarding the effectiveness of the ReLEX SMILE technique.

\begin{abstract}
Authors' contributions
CAN conceived the study, participated in surgeries, and contributed to writing the manuscript and design. DN participated in surgeries, made the analysis and interpretation of data. SDB helped to collect the data, made data curation and analysis and drafted the manuscript. AEB contributed to the study design and coordination. All authors read and approved the final manuscript.
\end{abstract}

\section{Funding}

Not applicable.

\section{Availability of data and materials}

The raw data analyzed during the current study are not publicly available but are available from the first author on reasonable request.

\section{Declarations}

\section{Ethics approval and consent to participate}

Data were retrospectively collected from the medical charts after approval of the study by the ethics Oculens Clinic Ethics Committee (IRB 2018-004). The study was conducted by respecting the Declaration of Helsinki and extensive explanations regarding the procedure, possible intraoperative and postoperative complications. Consent to participate was waived by the Ethics Committee.

\section{Consent for publication}

Not applicable.

\section{Competing interests}

The authors declare that there is no conflict of interest regarding the publication of this article.

\section{Author details}

'Department of Ophthalmology, "Iuliu Hatieganu" University of Medicine and Pharmacy, Victor Babeș Str., no. 8, 400012 Cluj-Napoca, Romania. ${ }^{2}$ Oculens Clinic, Calea Turzii, No. 134-136, 400501 Cluj-Napoca, Romania. ${ }^{3}$ Department of Medical Informatics and Biostatistics, "Iuliu Hațieganu" University of Medicine and Pharmacy, Louis Pasteur Str., no. 6, 400349 Cluj-Napoca, Romania. ${ }^{4}$ Department of Pathophysiology, "Iuliu Hațieganu" University of Medicine and Pharmacy, Victor Babeș Str., no. 8, 400012 Cluj-Napoca, Romania.

Received: 24 January 2021 Accepted: 29 November 2021

Published online: 08 December 2021

\section{References}

1. Pallikaris IG, Papatzanaki ME, Stathi EZ, Frenschock O, Georgiadis A. Laser in situ keratomileusis. Lasers Surg Med. 1990;10(5):463-8.

2. Shen Z, Shi K, Yu Y, Yu X, Lin Y, Yao K. Small incision lenticule extraction (SMILE) versus femtosecond laser-assisted in situ keratomileusis (FS-LASIK) for myopia:a systematic review and meta-analysis. PLoS One. 2016;11(7):e0158176.

3. Zhang Y, Shen Q, Jia Y, Zhou D, Zhou J. Clinical outcomes of SMILE and FS-LASIK used to treat myopia: a meta-analysis. J Refract Surg. 2016;32(4):256-65.
4. Sekundo W. SMILE, priniciples, techniques, complication, management and future concepts. 1st ed; 2015.

5. Kobashi H, Kamiya K, Shimizu K. Dry eye after small incision lenticule extraction and femtosecond laser-assisted LASIK: meta-analysis. Cornea. 2017:36(1):85-91.

6. Cai WT, Liu QY, Ren CD, et al. Dry eye and corneal sensitivity after small incision lenticule extraction and femtosecond laser-assisted in situ keratomileusus:a meta-analysis. Int J Ophthalmol. 2017;10(4):632-8.

7. Yan $\mathrm{H}$, Gong LY, Huang W, Peng YL. Clinical outcomes of small incision lenticule extraction versus femtosecond laser-assisted Lasik for myopia: a meta-analysis. Int J Ophthalmol. 2017;10(9):1436-45.

8. Sekundo W, Kunert KS, Blum M. Small incision corneal refractive surgery using the small incision lenticule extraction (SMILE) procedure for the correction of myopia and myopic astigmatism: results of a 6 month prospective study. Br J Ophthalmol. 2011;95:335-9.

9. Shah R, Shah $\mathrm{S}$, Sengupta $\mathrm{S}$. Results of small incision lenticule extraction: all-in-one femtosecond laser refractive surgery. J Cataract Refract Surg. 2011:37:127-37.

10. Hjortdal JO, Vestergaard AH, Ivarsen A, Ragunathan S, Asp S. Predictors for the outcome of small-incision lenticule extraction for myopia. J Refract Surg. 2012;28:865-71.

11. Kamiya K, Shimizu K, Igarashi A, Kobashi H. Visual and refractive outcomes of femtosecond lenticule extraction and small-incision lenticule extraction for myopia. Am J Ophthalmol. 2014;157:128-34.e2.

12. Ağca A, Demirok A, Cankaya Ki, Yaşa D, Demircan A, Yildirim Y, et al. Comparison of visual acuity and higher-order aberrations after femtosecond lenticule extraction and small-incision lenticule extraction. Cont Lens Anterior Eye. 2014;37:292-6.

13. Reinstein DZ, Archer TJ, Gobbe M. Small incision lenticule extraction (SMILE) history, fundamentals of a new refractive surgery technique and clinical outcomes. Eye Vis. 2014;1:3.

14. Vestergaard AH, Grauslund J, Ivarsen AR, Hjortdal JØ. Efficacy, safety, predictability, contrast sensitivity, and aberrations after femtosecond laser lenticule extraction. J Cataract Refract Surg. 2014;40(3):403-11.

15. Pedersen IB, Ivarsen A, Hjortdal J. Three-year results of small incision lenticule extraction for high myopia: Refractive outcomes and aberrations. J Refract Surg. 2015;31:719-24.

16. Chan TC, Ng AL, Cheng GP, Wang Z, Ye C, Woo VC, et al. Vector analysis of astigmatic correction after small-incision lenticule extraction and femtosecond-assisted LASIK for low to moderate myopic astigmatism. Br J Ophthalmol. 2016;100:553-9.

17. Wu W, Wang Y, Zhang H, Zhang J, Li H, Dou R, et al. One-year visual outcome of small incision lenticule extraction (SMILE) surgery in high myopic eyes: retrospective cohort study. BMJ Open. 2016;6:e10993.

18. Blum M, Täubig K, Gruhn C, Sekundo W, Kunert KS. Five-year results of small incision lenticule extraction (ReLEx SMILE). Br J Ophthalmol. 2016;100:1192-5.

19. Yıldırım Y, Alagöz C, Demir A, Ölçücü O, Özveren M, Ağca A, et al. Longterm results of small-incision lenticule extraction in high myopia. Turk J Ophthalmol. 2016;46:200-4.

20. Han T, Zheng K, Chen Y, Gao Y, He L, Zhou X, et al. Four-year observation of predictability and stability of small incision lenticule extraction. BMC Ophthalmol. 2016;16:149.

21. Liu YC, Chen Y, Wang D, Zhou Y, Zhang X, He J, et al. Clinical outcomes after SMILE and femtosecond laser-assisted LASIK for myopia and myopic astigmatism: a prospective randomized comparative study. Cornea. 2016;35(2):210-6

22. Ganesh S, Brar S, Relekar KJ. Epithelial thickness profile changes following small incision refractive lenticule extraction (SMILE) for myopia and myopic astigmatism. J Refract Surg. 2016;32:473-82.

23. Pietilä J, Huhtala A, Mäkinen P, Nättinen J, Rajala T, Salmenhaara K, et al. Uncorrected visual acuity, postoperative astigmatism, and dry eye symptoms are major determinants of patient satisfaction: a comparative, real-life study of femtosecond laser in situ keratomileusis and small incision lenticule extraction for myopia. Clin Ophthalmol. 2018;12:1741-55.

24. Ng ALK, Chan TCY, Cheng GPM, Jhanji V, Cong Y, Woo VCP, et al. Comparison of the early clinical outcomes between combined small-incision lenticule extraction and collagen cross-linking versus SMILE for myopia. J Ophthalmol. 2016;2016:2672980. 
25. Moshirfar M, Albarracin JC, Desautels JD, Birdsong OC, Linn SH, Hoopes PC Sr, et al. Ectasia following small-incision lenticule extraction (SMILE): a review of the literature. Clin Ophthalmol. 2017;11:1683-8.

26. Ivarsen A, Asp S, Hjordtal J. Safety and complications of more than 1500 small incision lenticule extraction pro- cedures. Ophthalmology. 2014;121:822-82.

27. Agca A, Tulu B, Yasa D, Yildirim Y, Yildiz B, Demirok A. Long-term (5 years) follow-up of small-incision lenticule extraction in mild-to-moderate myopia. J Cataract Refract Surg. 2019:45(4):421-6.

28. Reinstein DZ, Archer TJ, Gobbe M. Lenticule thickness readout for small incision lenticule extraction compared to Artemis three-dimensional very high-frequency digital ultrasound stromal measurements. J Refract Surg. 2014:30:304-9.

29. Titiyal JS, Rathi A, Kaur M, Falera R. AS-OCT as a rescue tool during difficult lenticule extraction in SMILE. J Refract Surg. 2017;33(5):352-4.

30. Titiyal JS, Kaur M, Rathi A, Falera R, Chaniyara M, Sharma N. Learning curve of small incision lenticule extraction: challenges and complications. Cornea. 2017;36(11):1377-82.

31. Titiyal JS, Kaur M, Shaikh F, Gagrani M, Brar AS, Rathi A. Small incision lenticule extraction (SMILE) techniques: patient selection and perspectives. Clin Ophthalmol. 2018;12:1685-99.

32. Shetty R, Negalur N, Shroff R, Deshpande K, Jayadev C. Cap lenticular adhesion during small incision lenticular extraction surgery :causative factors and outcomes. Asia Pac J Ophthalmol. 2017;6(3):233-7.

33. Jacob S, Agarwal A. White ring sign and sequential segmental terminal lenticular side cut dissection for uneventful and complete lenticular extraction in SMILE. J Refract Surg. 2018:34(2):140-1.

34. Ganesh S, Brar S, Arra RR. Refractive lenticule extraction small incision lenticule extraction: a new refractive paradigm. Indian J Ophthalmol. 2018;66(1):10-9.

35. Wang Y, Cui C, Li Z, Tao X, Zhang C, Zhang X, et al. Corneal ectasia 6.5 months after small-incision lenticule extraction. J Cataract Refract Surg. 2015:41:1100-6.

36. Sachdev G, Sachdev MS, Sachdev R, Gupta H. Unilateral corneal ectasia following small-incision lenticule extraction. J Cataract Refract Surg. 2015:41:2014-8

37. Ganesh S, Gupta R. Comparison of visual and refractive outcomes following femtosecond laser-assisted lasik with smile in patients with myopia or myopic astigmatism. J Refract Surg. 2014;30:590-6.

38. Kim JR, Hwang HB, Mun SJ, Chung YT, Kim HS. Efficacy, predictability, and safety of small incision lenticule extraction: 6-months prospective cohort study. BMC Ophthalmol. 2014;14:117.

39. Ang $\mathrm{M}, \mathrm{Ho} \mathrm{H}$, Fenwick $\mathrm{E}$, et al. Vision-related quality of life and visual outcomes after small-incision lenticule extraction and laser in situ keratomileusis. J Cataract Refract Surg. 2015;41:2136-44.

40. Luo J, Yao P, Li M, Xu G, Zhao J, Tian M, et al. Quantitative analysis of microdistortions in Bowman's layer using optical coherence tomography after SMILE among different myopic corrections. J Refract Surg. 2015;31:104-9.

41. Liu YC, Jayawinghe L, Ang HP, Lwin NC, Yam GH, Mehta JS. Effect of intraoperative corneal stromal pocket irrigation in small incision lenticule extraction. Biomed Res Int. 2015;2015:928608.

\section{Publisher's Note}

Springer Nature remains neutral with regard to jurisdictional claims in published maps and institutional affiliations.

Ready to submit your research? Choose BMC and benefit from:

- fast, convenient online submission

- thorough peer review by experienced researchers in your field

- rapid publication on acceptance

- support for research data, including large and complex data types

- gold Open Access which fosters wider collaboration and increased citations

- maximum visibility for your research: over $100 \mathrm{M}$ website views per year

At BMC, research is always in progress.

Learn more biomedcentral.com/submissions 